\title{
A cost-effective preventative approach to potentially save lives in the coronavirus pandemic, jointly using Vitamin D, Curcumin, and Vitamin C, (with updated dosage parameters).
}

\author{
Bakare, T A., a and Soar, J. S. ${ }^{b}$
}

Communication

\begin{abstract}
:
The current COVID-19 pandemic now believed to be based on the mutation of the SARS-CoV virus (first reported in 2002) to SARS-CoV-2 emerging in 2019 (with possibly further mutations), is naturally causing extreme worry and concern around the world with sometimes mixed and incoherent messages on how to deal with it. There is a plethora of information from previous epidemics caused by other coronaviruses such as severe acute respiratory syndrome, SARS (2002) and Middle East respiratory syndrome MERS (2012) from which we can extrapolate guidance on how to deal with the current pandemic. In the current absence of specific pharmaceutical agents, we propose assessing the extended tools that we already possess in our biological armoury to combat, prevent and control the spread of this virus. Using a set of precise criteria to locate such possible contenders, we conducted literature searches to find compounds that met these criteria. We have now reduced this to a shortlist of three agents that may be the best candidates. We propose vitamin C, vitamin D and Curcumin fit our criteria well as the biological properties summarised, further support their use. These compounds are widely available to the general public. They are available online and over-the-counter as supplements. Otherwise healthy individuals are, after taking some appropriate professional advice, safely able to selfadminister these agents as a prophylactic to protect themselves and to enhance their immune response. This would be especially desirable for the elderly and at-risk groups. These agents can also be used as adjunct therapy, particularly for those who may have early symptoms. This preventative therapy could be implemented whilst awaiting specific pharmaceutical drugs to emerge as a treatment for COVID-19. Our suggested compounds are a highly cost-effective way to potentially reduce the mortality that is regretfully mounting as a result of COVID-19 infection.
\end{abstract}

\section{INTRODUCTION:}

There have been several coronavirus (CoV)-related epidemics in our recent past, most notably severe acute respiratory syndrome (SARS)-CoV and Middle East respiratory syndrome (MERS)-CoV. The current COVID-19 pandemic caused by SARS-CoV-2 is another genus of the coronaviridae family which also include related zoonotic group $2 \mathrm{~b}$ or $2 \mathrm{c}$ Bat$\mathrm{CoV}[1]$.

The typical response to any epidemic is to turn to the pharmaceutical industry to produce a cure and to hopefully solve the problem as quickly as possible [2]. Within days of the current outbreak, for example, we experienced a clamour for both vaccines and antivirals to be made available almost instantaneously. Unfortunately, this narrow field approach means that other possible sources of immediate help are overlooked, potentially making it difficult to stem the extent and spread of the virus. We present here a theoretical re-appraisal of how we can make an impact on the SARS-Cov-2 pandemic immediately, by extending the mindset of what weaponry is at our disposal in a scientific, practical, sustainable and cost-effective way.

This means we co-examine all options including complementary approaches, we base our conclusions on fundamental science, particularly biochemistry and in this case immunology. Importantly, we also consider ethnographic, ecological, and environmental medical factors all together as an investigative continuum. This approach constitutes our standard conceptual protocol adopted by our institute which teaches the widest theoretical foundations of medicine and researches into new solutions for sustainable medicine.

Although there are dozens of biologically active putative substances we could use to augment the immune response that have been discussed in the literature, through our specific criteria review, we have reduced this to a shortlist of three compounds that may be the best candidates. The search criteria for this review is listed below. Using the prescribed list, we performed a literature search for safe complementary substances that seem to have the potential for reducing or even eliminating the severity of the SARS-Cov-2 virus infection and COVID-19 symptoms. 
The main criteria for searching are as follows:

- that the candidates are cheap

- they are easily available online or from local pharmaceutical outlets or through local retail shops

- that they are scientifically and clinically evidence driven

- have the potential to be highly efficacious

- that they do not harm.

\section{DISCUSSION:}

From our search findings, we forwarded three compounds in particular that we now propose.

\section{A. Vitamin D3 (1,25 Dihydroxycholicalciferol)}

The immune system defends and protects the body from foreign organisms and vitamin $\mathrm{D}$ has been demonstrated to play a very important role in modulating the innate and adaptive immune response. This it does via vitamin D receptors expressed on lymphocytes themselves. The active form of vitamin D (1,25-Dihydroxycholecalciferol finally converted in the kidneys) has numerous priming and modulatory effects on cells within the immune system. This includes inhibition of B cell lymphocyte proliferation which otherwise releases inflammatory cytokines, which can in excess produce harm. Also blockade of B cell differentiation, immunoglobulin secretion, suppression of $\mathrm{T}$ cell proliferation, of $\mathrm{T}$ cell maturation, with a skewing away from the inflammatory phenotype as well as the induction of T regulatory cells are all cellular responses known to be influenced by vitamin D [3].

These overall effects, resulting in decreased production of inflammatory cytokines (such as IL-17, IL-21), are accompanied by an increase in the production of anti-inflammatory cytokines (such as IL-10). Vitamin D also has effects on monocyte production of inflammatory cytokines (such as IL-1, IL-6, IL-8, IL-12 and TNF $\alpha$ ) [3]. This concert of immune responses by vitamin $\mathrm{D}$ is both remarkable and highly advantageous in the regulation of immune activity. These responses are known to be blunted with age, with immune deficiency situations, and within the elderly in particular [4].

In recent years, the clinical implications of vitamin D deficiencies on the immune system have become clear with studies showing an increased susceptibility to infections. This is documented in studies showing a high prevalence of both inadequate vitamin D intake, and serum level deficiency. This picture is repeated across Europe, particularly among some subgroups of the population such as those in institutions, those who are housebound, and non-Western immigrants. In fact, the prevalence of vitamin D deficiency, often defined as plasma levels of $1,25(\mathrm{OH}) 2 \mathrm{D} 3<25$ $\mathrm{nmol} / \mathrm{l}$, may be more common in populations with a higher proportion of at-risk groups, and/or that have low consumption of foods rich in vitamin D (naturally rich or fortified) and low use of vitamin D supplements[4].

It is therefore, a reasonable assumption to propose that the most at-risk individuals to succumb to the effects of coronavirus infection in Europe, including the UK, will be synonymous with those who are deficient in sufficient levels of vitamin D3.

As such we recommend individuals presenting with symptoms of COVID-19 should be given vitamin D3 as an adjunct therapy to increase vitamin D3 levels, thus boosting their immune response to COVID-19. In the US, the recommended dietary allowance (RDA) is the average daily level of intake sufficient to meet the nutrient requirements of $>97 \%$ healthy individuals which vary by age and gender. To date, the RDA by the Food and Nutrition Board is $400 \mathrm{IU}$ for children (0 - 12 months), 600 IU ( $1-70 \mathrm{y} / \mathrm{o})$, and $800 \mathrm{IU}$ (>71 years of age) [5]

Also, in the UK, the government has been advised by Public Health England (PHE) based on the recommendations of the Scientific Advisory Committee on Nutrition (SACN), that vitamin D equivalent to an average daily intake of 400 IU [10 micrograms]) throughout the year, including in the winter months is recommended to protect bone and muscle health [6]

However, this recommendation is not a widely shared consensus as the guideline addressed needs on a population and public health level but not treatment of specific medical conditions. The safe upper limit was raised from 2,000 IU daily to 4,000 IU for adults, and declares a safe upper limit of 1,000 to 3,000 IU per day in children depending on their age. It has been demonstrated that overweight and obese individuals may require higher daily intake as much as up to 8,000 IU per day. In fact, some of the participants took daily doses of vitamin $\mathrm{D}_{3}$ as high as 15,000 IU without any untoward toxicity [7] 
A typical adult formulation can be as high as $5-10,000 \mathrm{IU}(0.125 \mathrm{mg}-0.25 \mathrm{mg})$ in some regions of the world. This can be taken orally in the form of supplements presented as a liquid in capsule form, rather than the calcium bound tablet that may affect cardiovascular activity and blood pressure. However, recognising that different countries have different upper limits, the user is strongly advised to seek their respective countries medical advice before setting final upper levels. A daily recommended dietary allowance (RDA) ranges from $500-1000 \mathrm{IU} /$ day has been a typical benchmark used by clinical nutritionists and medical practitioners as an adequate measure to address vitamin D deficiency.

Toxic intake levels are very much higher than this, stated to be around 40,000 IU per day over a prolonged period of intake at such levels [4]. These much higher levels should be under strict medical supervision only. Also, studies of other disease populations have suggested doses of vitamin $\mathrm{D}_{3}$ of $50,000-100,000 \mathrm{IU} /$ week are tolerable without any demonstrable adverse effects [8]

\section{B. The next potential bio-active compound that met our search criteria is Vitamin C (Ascorbic acid).}

Vitamin $\mathrm{C}$ (which is not manufactured in humans) has to be taken in the diet. It is absorbed in the small intestine through both diffusion and active transport. In the US, the RDA for age 4 to adults is $90 \mathrm{mg} / \mathrm{day}$ [9]. In the UK, the government recommendation for vitamin $C$ for adolescents to $>75$ years is $40 \mathrm{mg} /$ day [10] Dietary doses of up to 100 $\mathrm{mg} / \mathrm{day}$ are almost completely absorbed. The highest concentrations of ascorbic acid are in the pituitary gland, adrenal gland, the brain, leukocytes, and eyes. The limited absorption can necessitate intravenous introduction in acute cases.

Ascorbic acid functions as a cofactor, enzyme complement, co-substrate, and a powerful anti-oxidant in a variety of reactions and metabolic processes. It is important in collagen synthesis and fibroblast linkage. It also stabilises vitamin $\mathrm{E}$ and folic acid, and enhances iron absorption, to mention a few. It neutralises free radicals and toxins as well as attenuating the inflammatory response.

It is an essential part of human nutrition, playing many important roles as a potent antioxidant as well as contributing to both the innate and adaptive immune system. In terms of our ability to effectively combat infections, deficiency of vitamin $\mathrm{C}$ has been shown to enhance differentiation and proliferation of lymphocytes which are required for effective defence against viral infections.

Furthermore, in the context of the respiratory symptoms caused by coronavirus, vitamin C supplementation appears to prevent and strongly moderate respiratory infections in the elderly group. The current information from China and Italy shows that elderly people are particularly susceptible to the devastating effects of COVID-19 infection. This is due to immunosenescence and decreased immune cell function in this age group. Common viral infections such as respiratory illnesses that are usually self-limiting in healthy young people, can lead to the development of complications such as pneumonia, resulting in increased morbidity and mortality in elderly people. We are observing this in this current pandemic.

It has been shown that there is a lower mean vitamin $\mathrm{C}$ status in both independent living and institutionalised elderly people, indicated by lowered plasma and leukocyte concentrations [4,11], which is of concern because low vitamin C concentrations $(<17 \mu \mathrm{mol} / 1)$ in older people (aged 75-82 years) are strongly predictive of all-cause mortality [12]. Epidemiological studies have indicated that hypovitaminosis $\mathrm{C}$ (plasma vitamin $\mathrm{C}<23 \mu \mathrm{mol} / \mathrm{l}$ ) is relatively common in Western populations, and vitamin C deficiency $(<11 \mu \mathrm{mol} / \mathrm{l})$ is the fourth leading nutrient deficiency in the United States $[\mathbf{1 3 , 1 4}]$. Acute and chronic diseases are also prevalent in this age group and this may also play an important part in the reduction of vitamin $\mathrm{C}$ reserves $[\mathbf{1 5}, \mathbf{1 6}]$. Institutionalisation is an additional aggravating factor in this age group, resulting in even lower plasma vitamin C levels than in non-institutionalised elderly people. It has been shown that elderly hospitalised patients with acute respiratory infections have improved significantly better with vitamin $\mathrm{C}$ supplementation than those not receiving the vitamin [17]. Placebo-controlled trials have shown quite consistently that the duration and severity of common colds are reduced in the vitamin C groups, further indicating that viral respiratory infections in humans are effectively decreased. In particular, three controlled trials with human subjects reported a significantly lower incidence of pneumonia with vitamin $C$ supplement $[\mathbf{1 8 , 1 9 ]}$. We are aware of some reports which suggest these effects are marginal and other which suggest that intravenous vitamin $\mathrm{C}$ has a particularly effective response.

The possibility of vitamin $\mathrm{C}$ contributing to a strong ameliorating effect on viral based respiratory tract infections is understood to be prevalent. Hence, in the current absence of a specific treatment for SARS-Cov-2, we recommend the addition of vitamin C of about $100-200 \mathrm{mg} /$ day to the daily dietary intake as essential for prophylactic prevention and treatment of COVID-19 infection. This dose appears to provide adequate saturating plasma concentrations in healthy individuals and is, therefore, anticipated to cover requirements for the reduction of many infection risks including the current respiratory infection by COVID-19 [20].

C. The third potential therapeutic agent that meets our search criteria is the compound Curcumin. 
This molecule (part of the curcuminoid family) is the principal curcuminoid of turmeric (Curcuma longa), which is commonly used as a spice in cooking and a yellow pigment in the food processing industry. Curcumin is generally recognized as safe (GRAS) by the FDA as a nutrient in food and is, therefore, exempt from the requirement of premarket approval [21]. Recent studies have demonstrated that curcumin has a variety of biological and pharmacological actions providing a wide range of protection and promotion of human health [22].

It is now known that SARS-Cov-2 is also an enveloped virus and, as such, our search criteria specifically included natural remedies with antiviral properties against enveloped virus strains. We found in vitro studies have positively demonstrated effective antiviral properties of curcumin against enveloped viruses such as Dengue virus (DENV), influenza virus and emerging arboviruses like the Zika virus (ZIKV) or chikungunya virus (CHIKV) [23,24,25,26]. The beneficial effect of curcumin in humans is based on clinical trials that have been conducted in the past few years; a search at clinicaltrials.gov reveals 76 completed clinical studies using curcumin for a variety of conditions such as colon, lung, and breast cancer, as well as inflammatory bowel disease.

A review of the properties of curcuminoids both alone or in modified form, found anti-inflammatory, antimicrobial, antiviral, anti-tumour and anti-immunomodulatory properties (preventing the attenuation of the immune response). These studies suggest that curcuminoids have a very significant role to play in fundamental disease control at the cellular level [27]. Dietary turmeric supplements with varying formulations are available in the global market and have been used in multiple studies [28]. Curcumin is usually taken in food as turmeric but is not well absorbed when taken alone. However, its bioavailability is increased when combined with piperine (a black pepper extract) or when combined with lipids (as with commercial curcumin-based preparations such as BCM-95 ${ }^{\circledR}$ or Meriva ${ }^{\circledR}$ ).

A re-evaluation of curcumin as a food additive by a panel on food additives and nutrient sources concluded that the present database supports the use of curcumin at an acceptable daily intake of $3 \mathrm{mg} / \mathrm{kg} /$ day [29]. To supplement curcumin with piperine, $500 \mathrm{mg}$ of the former with $20 \mathrm{mg}$ of the latter, thrice a day (i.e., 1,500 $\mathrm{mg}$ of curcumin and $60 \mathrm{mg}$ of piperine per day) is recommended. To supplement BCM-95 ${ }^{\circledR}$, a patented combination of curcumin and essential oils, $500 \mathrm{mg}$ twice a day (i.e., 1,000 mg/day) is recommended. To supplement $\mathrm{Meriva}^{\circledR}$, a patented combination of curcumin and soy lecithin, 200-500 $\mathrm{mg}$ twice a day (i.e., 400-1,000 mg/day). (https://examine.com/supplements/curcumin/) [30,31,32].

In some clinical trials, curcumin was used alone or in combination with other agents and delivery systems such as liposomal encapsulation. A comprehensive review of the potential health benefits of curcumin is provided by Xia-Yu $\mathrm{Xu}$ et al [33].

Although, there is still some debate on the extent of clinical beneficial effects (and challenges) regarding curcumin as a therapeutic agent, there is abundant experimental evidence to show that it is a highly promising immune defence intermediate derived from an increasingly common functional food. It has strong and broad efficacy in preventing certain diseases, thus, combining the findings from in vitro studies and clinical trials, we are able to suggest its use as a safe agent in the current context of COVID-19.

\section{CONCLUSION:}

It is important to acknowledge the efforts by clinical pharmaceutical researchers to find the most effective drugs against COVID-19 either by re-purposing known antivirals or discovering new ones. In fact, there are a number of promising findings that are being currently evaluated such as Azithromycin (antibiotic) added to hydroxychloroquine (an antimalarial agent), which has purportedly shown efficiency in eradicating the SAR-Cov-2 virus within 3-6 days [34]. although yet to be demonstrated in a clinical trial. A recent animal study (in mouse) has demonstrated the potential of another agent, EIDD-2801 showing a broad spectrum antiviral activity against SARS-CoV- 2, MERS-CoV, SARS-CoV, and related zoonotic group $2 \mathrm{~b}$ or $2 \mathrm{c}$ Bat-CoVs, as well as increased potency against a coronavirus bearing resistance mutations to a nucleoside analogue inhibitor. Notably, Favilavir (aka. Favipiravir), an RNA polymerase inhibitor antiviral drug has been approved for clinical trials in China as a repurposed antiviral drug for treating COVID-19 at the point of writing this paper (https://www.clinicaltrialsarena.com/analysis/coronavirus-mers-cov-drugs/).

We believe that all these avenues are important and should be given urgent consideration however, there may well be considerable delays in any of these contenders reaching the general bedside. Aside from the clinical desirability, there are mounting commercial and political considerations to any drug successfully reaching the patient. This is why we strongly believe that our argument presented here may be a valuable contribution as early defence, namely that the suggested selected regime is immediately usable, promises to be very efficacious, and is cheaply available. It also offers 
bof SARS-Cov-2. Should any new pharmaceutical drug contender reach patients, our regime still has validity as adjunct therapy.

However, it is likely that we are still some time away from concluding satisfactory clinical trials of these potential drugs, hence we believe using well understood bioactive molecules that are easily available to the public would help stem the increase in COVID-19 cases and moderate potential deaths.

A new UK analysis from University College London (UCL), which estimates the excess number of deaths caused by COVID-19 in relation to underlying medical conditions and age, using NHS health records from 3.8 million adults in England has been forwarded. The study suggests that the current strategy of slowing the spread of coronavirus could lead to at least 35,000 and as many as 70,000 excess deaths over one year [35]. We believe our regime can reduce this number for both the elderly population and the at-risk groups who are most susceptible to the severe manifestations of COVID-19 disease.

The important consideration is to start the triple regime as soon as possible and certainly on experiencing any type of symptom associated with the coronavirus, and certainly if even mild respiratory symptoms commonly described as flulike with fever, rhinorrhea, coughing and intermittent shortness of breath are experienced. It is not unreasonable to take the biological agents as prophylaxis, since very few known contraindications are present at the dosages suggested. As with any drugs and/or supplements, a general understanding of the patient's clinical history, comorbidities, allergies, and drug interactions, should be appraised before use of any one or all three compounds. Consulting a healthcare professional is advised to avoid triggering adverse effects of hypervitaminosis. This could include raised blood pressure or imbalance in kidney function associated with introducing Vitamin D, gastrointestinal issues and blood thinning possibilities with Curcumin, and sickle cell disease and haemochromatosis issues with Vitamin C, all rare but possible reactions. We do however believe that self-administering the above in suggested dosages at the recommended daily intake (RDI) levels only, if otherwise healthy, and in parallel with contacting a professional health adviser first, presents little risk and could be life-saving and cost-effective for the NHS overall. Revision (possibly upwards) of vitamin D (or indeed Vit $\mathrm{C}$ and curcumin) intake may then follow after more thorough medical advice for patients presenting with mild to moderate symptoms and not requiring hospitalization. The regime could effectively moderate or prevent the progression to more severe respiratory tract symptoms of coronavirus infection which are to be avoided at all costs. The RDI dosages suggested apply to otherwise healthy individuals. However, states of absorption of these compounds also differ such that, even in mild disease compromised states, the serum level requirements of these compounds may differ. This would be a subject for close medical supervision only.

\section{Footnote:}

As of 15th April 2020, the number of coronavirus cases reported in India is 12,322 with a mortality figure of 405 , against a population of 1.38 billion. This is correspondingly very low indeed. Although a number of factors could be masking truer statistics, India has invariably escaped the sort of casualty figures for similar coronavirus infections (SARS, MERS, etc) that other countries have endured in the past.

However, there is also a possibility that this relatively low number of positive cases and low mortality could be a reflection of the high levels of dietary intake of rich natural sources of vitamin C (e.g., chilli peppers, yellow sweet peppers and a largely vegetarian diet), high levels of curcumin (from turmeric) and having adequate levels of vitamin D from exposure to sunlight from the hot climate. India is the highest turmeric consuming country in the world.

Author Details:

a. Dr T.A. Bakare., Clinician scientist, Viapath, Guy’s Hospital, London

b. Dr J S Soar., Director of Studies and Research. The School of Health and Neural Sciences (SOHANS), Nottingham, (UK).

All enquiries to sohansmed@gmail.com

\section{REFERENCES}

1. https://www.ecdc.europa.eu/en/factsheet-health-professionals-coronaviruses

2. Timothy P. Sheahan, Amy C. Sims, Shuntai Zhou, et al An orally bioavailable broad-spectrum antiviral inhibits SARS-CoV-2 and multiple endemic, epidemic and bat coronavirus doi: https://doi.org/10.1101/2020.03.19.997890 
3. Aranow C, Vitamin D and the Immune System. J Investig Med. 2011 Aug; 59(6): 881-886.

4. Spiro A, Buttriss JL. Vitamin D: An overview of vitamin D status and intake in Europe. Nutr Bull. 2014 Dec;39(4):322-350.

5. Ross AC, Taylor CL, Yaktine AL, Del Valle HB, eds. Institute of Medicine (US) Committee to Review Dietary Reference Intakes for Vitamin D and Calcium, 2011 Dietary Reference Intakes for Calcium and Vitamin D. Washington (DC): National Academies Press (US)

6. The Scientific Advisory Committee on Nutrition (SACN) vitamin D and health report (2016)

7. Kimball S. M, Mirhosseini N \& Holick M.F (2017) Evaluation of vitamin D3 intakes up to 15,000 international units/day and serum 25-hydroxyvitamin D concentrations up to $300 \mathrm{nmol} / \mathrm{L}$ on calcium metabolism in a community setting, Dermato-Endocrinology, 9:1, DOI: 10.1080/19381980.2017.1300213]

8. Jetty V, Glueck CJ, Wang P, et al. Safety of 50,000-100,000 Units of Vitamin D3/Week in Vitamin D-Deficient, Hypercholesterolemic Patients with Reversible Statin Intolerance. N Am J Med Sci. 2016;8(3):156-162. doi:10.4103/1947-2714.179133

9. Institute of Medicine. Food and Nutrition Board. Dietary Reference Intakes for Vitamin C, Vitamin E, Selenium, and Carotenoids. Washington, DC: National Academy Press, 2000.

10. Public Health England (PHE) publications gateway number: 2016202

11. Simon JA, Hudes ES, Tice JA. Relation of serum ascorbic acid to mortality among US adults. J Am Coll Nutr. 2001 Jun; 20(3):255-63.

12. Fletcher A., Breeze E., ShettBy P. Antioxidant vitamins and mortality in older persons: Findings from the nutrition add-on study to the Medical Research Council Trial of Assessment and Management of Older People in the Community. Am. J. Clin. Nutr. 2003; 78:999-1010.

13. Schleicher R.L., Carroll M.D., Ford E.S., Lacher D.A. Serum vitamin C and the prevalence of vitamin C deficiency in the United States: 2003-2004 National Health and Nutrition Examination Survey (NHANES) Am. J. Clin. Nutr. 2009; 90:1252-1263.

14. Thurman J., Mooradian A. Vitamin supplementation therapy in the elderly. Drugs Aging. 1997; 11:433-449.

15. Hanck A. Vitamin C in the elderly. Int. J. Vitam. Nutr. Res. Suppl. 1983; 24:257-269

16. Schorah C.J. The level of vitamin C reserves required in man: Towards a solution to the controversy. Proc. Nutr. Soc. 1981; 40:147-154.

17. Hunt C., Chakravorty N., Annan G. The clinical and biochemical effects of vitamin C supplementation in short-stay hospitalized geriatric patients. Int. J. Vitam. Nutr. Res. 1984; 54:65-74.

18. Hemilä H. Vitamin C and Infections. Nutrients. 2017; 9(4):339.

19. Levine M., Dhariwal K.R., Welch R.W., Wang Y., Park J.B. Determination of optimal vitamin C requirements in humans. Am. J. Clin. Nutr. 1995; 62:1347S-1356S.

20. Carr A.C., Frei B. Toward a new recommended dietary allowance for vitamin $\mathrm{C}$ based on antioxidant and health effects in humans. Am. J. Clin. Nutr. 1999; 69:1086-1087.

21. USFDA GRAS Notice 822. Curcumin (2018) https://www.curcuminoids.com/gras-affirmed-usfda 
22. Praditya D, Kirchhoff L, Brüning J, Rachmawati H, Steinmann J, Steinmann E. (2019) Anti-infective Properties of the Golden Spice Curcumin. Front. Microbiol., O3 May 2019| https://doi.org/10.3389/fmicb.2019.00912

23. Chen TY et al. Inhibition of enveloped viruses infectivity by curcumin PLoS One. 2013; 8(5): e62482;

24. Balasubramaniam A et al,;Antiviral Res. 2019 Feb; 162: 71-78

25. Tzu-Yen Chen, Da-Yuan Chen, Hsiao-Wei Wen, Jun-Lin Ou, Shyan-Song Chiou, Jo-Mei Chen, Min-Liang Wong, Wei-Li Hsu Inhibition of enveloped viruses infectivity by curcumin PLoS One. 2013; 8(5): e62482

26. Mounce BC, Cesaro T, Carrau L, Vallet T, Vignuzzi M. Curcumin inhibits Zika and chikungunya virus infection by inhibiting cell binding. Antiviral Res. 2017 Jun;142: 148-157.

27. Greil R, Greil-Ressler S, Weiss L, et al. A phase 1 dose-escalation study on the safety, tolerability and activity of liposomal curcumin $\left(\right.$ Lipocurc $^{\mathrm{TM}}$ ) in patients with locally advanced or metastatic cancer. Cancer Chemother Pharmacol. 2018;82(4):695-706.

28. Amalraj A. Biological activities of curcuminoids, other biomolecules from turmeric and their derivatives-A review. J. Tradit. Complement. Med. 2017; 7:205-23

29. Scientific Opinion on the Re-evaluation of curcumin (E100) as a food additive, EFSA 2010; 8(9):1679.

30. Kunnumakkara A.B., Br. J. Pharmacol. 2017; 174:1325-1348

31. Hussain Z, Colloids Surf. B Biointerfaces. 2017; 150:223-241.

32. Mirzaei H., Biomed. Pharmacother. 2017; b85:102-112.

33. Xu, Xiao-Yu et al. "Bioactivity, Health Benefits, and Related Molecular Mechanisms of Curcumin: Current Progress, Challenges, and Perspectives.” Nutrients vol. 10,10 1553. 19 Oct. 2018)

34. Philippe Gautret ,Jean-Christophe Lagier ,Philippe Parola ,Van Thuan Hoang ,Line Meddeb ,Morgane Mailhe ,Barbara Doudier ,Johan Courjon ,Val' erie Giordanengo ,Vera Esteves Vieira ,Herv' e Tissot Dupont ,St' 'ephane Honor'e ,Philippe Colson ,Eric Chabri'ere ,Bernard La Scola ,Jean-Marc Rolain ,Philippe Brouqui ,Didier Raoult, Hydroxychloroquine and azithromycin as a treatment of COVID-19: results of an openlabel non-randomized clinical trial,International Journal of Antimicrobial Agents(2020), https://doi.org/10.1016/j.ijantimicag.2020.105949

35. Denaxas, Spiros \& Hemingway, Harry \& Shallcross, Laura \& Noursadeghi, Mahdad \& Williams, Bryan \& Pillay, Deenan \& Pasea, Laura \& González-Izquierdo, Arturo \& pagel, christina \& Harris, Steve \& Torralbo, Ana \& Langenberg, Claudia \& Wong, Wai \& Banerjee, Amitava. (2020). Estimating excess 1- year mortality from COVID-19 according to underlying conditions and age in England: a rapid analysis using NHS health records in 3.8 million adults. 10.13140/RG.2.2.36151.27047.

Both authors TAB and JSS declare no competing interests.

V5.8b (3):V6.1d 